\title{
Les besoins des patients/familles qui subissent une greffe autologue de cellules souches du sang périphérique
}

par Betty Ann Griffiths

\section{Résumé}

La greffe autologue de cellules souches du sang périphérique (GACSSP) en tant que thérapie de récupération après une chimiothérapie myélosuppressive est devenue la norme dans le traitement de plusieurs types de tumeurs malignes. La plupart des traitements pré-greffe ont lieu dans des cliniques externes d'oncologie, et l'utilisation de facteurs de croissance hématopoïétique accélère l'octroi du congé après l'intervention de greffe. Les patients/familles sont alors tenus d'assurer eux-mêmes la gestion de soins complexes dans le milieu familial (Johns, 1998; Poloquin, 1997; Schulmeister et al., 2005). On a entrepris cette évaluation des besoins en vue de dégager des façons d'améliorer la qualité de la prestation de soins aux patients/familles qui subissent une GACSSP. Dans le cadre de cette recherche qualitative, on a utilisé aux fins de collecte de données des entretiens libres, l'observation et les notes prises sur le terrain. Les données ont été analysées au moyen d'analyses thématiques du contenu. Il s'en est dégagé quatre concepts clés pour l'amélioration de la qualité de la prestation des soins : les connaissances, la communication, le soutien et les compétences.

\section{Renseignements généraux}

La greffe autologue de cellules souches du sang périphérique (GACSSP) s'utilise de plus en plus fréquemment pour traiter le lymphome non hodgkinien, la maladie de Hodgkin, le myélome multiple et le cancer du sein (Johns, 1998; Poloquin, 1997; Schulmeister, Quiett et Mayer, 2005). Cette intervention implique le prélèvement de cellules souches en circulation dans le sang périphérique au moyen d'un processus de collecte de cellules souches. On fait alors subir à ces dernières un traitement de chimiothérapie à forte dose et, dans des cas particuliers, un traitement de radiothérapie. À la suite de ces traitements, le sang est réinjecté dans le système sanguin du patient. Les objectifs de la GACSSP sont soit de guérir soit de prolonger le temps de survie sans maladie (Coleman, Coon, Mattox et O'Sullivan, 2002; Johns, 1998; Poloquin, 1997).

Dans le passé, la gestion du traitement préalable à une GACSSP et des soins intensifs qui l'accompagnent, les soins post-greffe complexes et les soins de suivi étaient tous assurés dans des centres de soins tertiaires. Toutefois, il existe un nombre restreint de centres de soins tertiaires qui offrent cette modalité de traitement, et le nombre de patients dirigés pour un tel traitement est en hausse. Les progrès au niveau de la technologie des cellules souches et l'utilisation de facteurs de croissance hématopoïétique facilitent le traitement pré-greffe dans les cliniques externes d'oncologie et accélèrent l'octroi du congé après l'intervention. Les coûts toujours croissants des soins de santé contribuent à la pratique des congés rapides. Ces changements ont mené au besoin d'exiger du patient et des aidants naturels à la fois qu'ils assument la responsabilité de l'évaluation, des soins directs, de l'administration des médicaments, de l'entretien des lignes intraveineuses centrales, du changement des pansements et du soutien affectif dans le milieu familial (Hurley, 1997; Schulmeister, Quiett et Mayer, 2005). Les infirmières des centres de soins oncologiques d'orientation et les infirmières visiteuses en santé communautaire sont par conséquent tenues d'assumer plus de responsabilités en ce qui a trait aux soins pré-greffe, aux soins complexes et aux soins de soutien postgreffe. Ces infirmières doivent être prêtes à répondre aux besoins de la population en matière de renseignements et de soutien pratique ainsi qu'à ses besoins physiques, psychosociaux et spirituels (Hurley, 1997; Johns, 1998; Kemp et Dickerson, 2002). Du point de vue du patient et de la famille, on en sait peu au sujet de la qualité des soins liés aux GACSSP offerts en clinique externe ou au sujet de la satisfaction des patients par rapport à ces soins (Schulmeister, Quiett et Mayer, 2005).

\section{Objectif}

L'objectif de cette évaluation des besoins était de déterminer la façon dont des intervenants en soins de santé spécialisés en oncologie peuvent contribuer à l'amélioration de la qualité des soins aux patients/familles qui subissent une GACSSP dans leur propre collectivité, du moment de l'orientation à la période suivant la greffe. La recherche a été motivée avant tout par le fait que deux infirmières du centre d'orientation (a) savaient que la GACSSP est un traitement de plus en plus courant, (b) se sentaient mal préparées à répondre aux besoins de cette population et (c) étaient par conséquent incapables de se conformer aux normes de la pratique en soins infirmiers en oncologie (ACIO, 2001).

\section{Questions de recherche}

Quels sont les besoins particuliers des patients/familles qui subissent une greffe autologue de cellules souches du sang périphérique?

Quelles interventions infirmières répondent aux besoins des patients/familles qui subissent une GACSSP dans la collectivité?

\section{Méthodologie}

Devis

Cette recherche descriptive qualitative était fondée sur une approche phénoménologique. On a utilisé l'observation, les notes prises sur le terrain et des entretiens non structurés pour recueillir les données.

Contexte

Le contexte comprenait les milieux naturalistes des diverses zones où avaient lieu les GACSSP dans les centres de soins tertiaires, notamment la clinique externe d'oncologie, l'aire de collecte des cellules souches, le service d'hématologie et la zone de radiothérapie. On a interviewé une des patientes chez elle, et on a interviewé une des infirmières au téléphone à son travail.

\section{Échantillon}

Parmi les participants, on compte un échantillon de convenance d'infirmières, de patients et de membres de la famille que l'on a estimés être représentatifs de la population en question. Les patients et les membres de la famille participaient activement à une des phases du processus de greffe (consultation aux fins d'admissibilité, chimiothérapie, collecte de cellules souches, radiothérapie, avant ou

Lorsqu'elle a écrit cet article, Betty Ann Griffiths était une étudiante de second cycle en sciences infirmières au D'Youville College, à Buffalo, New York. Depuis, elle a obtenu son diplôme de maîtrise de ce même collège. Elle travaille actuellement au Juravinski Cancer Centre de Hamilton, à titre d'infirmière en pratique avancée en soins de soutien. Elle peut être jointe à grifiths@vaxxine.com. 
après la greffe); les infirmières participaient à la prestation de soins aux patients/familles. Les participants de l'étude ont été choisis dans un centre de soins tertiaires, dans un centre d'orientation et dans une collectivité associée; la plupart des participants, cependant, provenaient du centre de soins tertiaires. La population de l'échantillon $(\mathrm{N}=24)$ comprenait des patients $(\mathrm{N}=11)$, des membres de la famille de patients $(\mathrm{N}=5)$ et des infirmières $(\mathrm{N}=8)$. L'échantillon de patients comprenait sept femmes et quatre hommes ayant entre 19 et 60 ans. Les diagnostics de cancer primitif variaient : maladie de Hodgkin, lymphome, leucémie, cancer du sein et deux cas dans lesquels les chercheuses ne connaissaient pas le diagnostic. La capacité fonctionnelle variait de zéro à trois selon le stade d'avancement de la maladie et le traitement. Deux patients subissaient une greffe autologue de la moelle osseuse. Tous les autres patients étaient admissibles à subir une GACSSP ou en subissaient déjà une. Les patients étaient orientés par diverses régions du Sud de l'Ontario ou d'autres provinces. Parmi les participants des familles, on comptait deux parents, un fils adulte, une épouse et un époux. Les âges oscillaient entre 30 et 50 ans. Tous les participants avaient un membre de la famille (fils adolescent, mère, épouse ou époux) qui en était à une phase ou une autre du processus de greffe. Les infirmières participant à l'étude étaient autorisées et travaillaient à temps plein. Deux des infirmières avaient un baccalauréat en sciences infirmières. On ne connaissait pas les antécédents pédagogiques des autres infirmières. Le nombre d'années d'expérience avec les greffes variait entre trois et treize ans, avec une médiane de sept.

\section{Collecte des données}

Les données ont été recueillies par deux étudiantes-chercheuses lors d'un placement clinique entre janvier et mars 1997, pour une durée totale de 96 heures. Les chercheuses ont collecté les données pendant la prestation directe de soins aux patients, pendant l'enseignement et le soutien aux familles et durant les interactions avec les infirmières tout au long de la journée. Les chercheuses ont interviewé les participants soit ensemble, soit chacune de leur côté. Les deux chercheuses avaient plusieurs années d'expérience de pratique clinique en oncologie, ce qui a contribué à la qualité des observations, de la communication et des aptitudes de counselling. De plus, les deux infirmières avaient également complété un cours avancé d'aptitudes de communication et avaient exercé leurs compétences avec des modèles normalisés de patients, dans le cadre du Programme de soins en oncologie destinés aux adultes offert par l'Université McMaster. Berg (1998) met l'accent sur le besoin de solides aptitudes d'observation, d'écoute et de communication pour la collecte de données.

Tous les participants ont été informés des antécédents des chercheuses dans le domaine de l'oncologie clinique et de leur participation au Programme de soins en oncologie destinés aux adultes. On a expliqué à chaque participant le but et les objectifs de l'évaluation des besoins et le caractère bénévole de la recherche, en mettant l'accent sur leur droit de se retirer des discussions ou des dialogues en tout temps s'ils ressentaient souffrance ou inconfort. La vaste expérience clinique des chercheuses des soins infirmiers en oncologie leur a fourni (a) une expertise qui leur a permis d'aborder les questions affectives éprouvées par les participants et (b) la capacité d'orienter les participants vers des précepteurs ou d'autres ressources appropriées, au besoin. L'anonymat a été assuré en n'identifiant ni les participants ni le centre de greffe. On a obtenu le consentement verbal de tous les participants. Selon Berg (1998), lorsque le consentement n'est pas obtenu par écrit, les réponses affirmatives et la participation à des entrevues constituent un consentement implicite. De plus, l'anonymat des participants est un des avantages de ce type de consentement éclairé (Berg, 1998). En vue d'assurer la légitimité des méthodes de recherche et d'analyse, on a fait retenu les services d'un professeur d'université à titre de consultant.

Les données démographiques recueillies variaient en fonction de la catégorie du participant. Le statut socioéconomique et les données liées à la scolarité n'étaient généralement pas compris dans l'étude, bien que les chercheuses reconnaissaient que ces variables ont une incidence sur la cognition et le comportement. Les données recueillies auprès de l'échantillon de patients comprenaient l'âge, le sexe, le diagnostic, la capacité fonctionnelle, le type de greffe, le centre d'orientation et la perception qu'avait le patient du soutien qu'il recevait. On a demandé aux participants à l'étude de partager avec les chercheuses leurs expériences personnelles du processus de greffe, par le biais d'un compte rendu. Chaque participant déterminait le degré de détail fourni dans sa narration. Toutes les expériences des participants ont été transcrites et présentées sous forme de rapport de la façon la plus fidèle possible. Les chercheuses ont utilisé des questions d'approfondissement afin d'obtenir plus de détails. «Quand avez-vous ressenti le plus d'angoisse ou de nervosité? » et « En quoi les infirmières auraient-elles pu offrir plus de soutien? » sont des exemples de questions utilisées pour obtenir plus de détails ou pour clarifier certains renseignements. On a utilisé des questions fermées principalement avec les infirmières participantes. "Combien de temps, en moyenne, est consacré à l'enseignement et au soutien aux patients/familles? », « À quels documents les patients/familles ont-ils accès? » et « Selon vous, quel est le plus important changement qu'il conviendrait d'apporter en vue d'améliorer les soins aux patients/familles qui subissent une GACSSP? » sont des exemples des questions posées aux infirmières.

Dans la plupart des cas, les chercheuses ont réalisé les entrevues et recueilli leurs observations simultanément. Chaque chercheuse a pris des notes individuelles en se basant sur ses propres observations et interprétations. Elles ont transcrit le plus précisément possible les interactions, préoccupations, questions et émotions des participants, de même que les événements vécus. Elles prenaient des notes à différents moments de la journée, selon la situation. La plupart du temps, elles consignaient chaque jour un minimum de renseignements immédiatement après une rencontre avec un participant et complétaient les détails en fin de journée. Une fois que le processus a été lancé, elles ont pris très peu de notes sur le terrain. Les dialogues interactifs, lorsqu'ils n'étaient pas immédiatement transcrits, étaient reconstitués par les deux chercheuses après réflexion. La consignation de citations directes posait parfois problème lorsqu'il y avait un délai entre l'entretien et la prise de notes. Polit et Hungler (1997) signalent que de tels délais peuvent mener à des trous de mémoire. Toutefois, Berg (1998) soutient que même s'il est impossible de consigner précisément chaque nuance des comportements, conversations et événements au cours d'une évaluation sur le terrain, il est possible de produire des notes détaillées et très précises. Selon les chercheuses, il était impossible de participer activement à l'observation et à l'écoute et de manifester une sollicitude réelle tout en prenant des notes.

\section{Analyse des données}

Les données ont été analysées au moyen d'une analyse du contenu permettant de cerner les thèmes récurrents. L'analyse a été réalisée par deux examinatrices indépendantes après la collecte des données. Sept thèmes récurrents se sont dégagés dans les groupes des patients et des familles. Les thèmes sont appuyés de commentaires des participants.

Thème un : Un impact psychosocial/spirituel considérable tout au cours du processus de greffe

Les patients et les membres de leur famille ont fait part de leurs sentiments de peur : peur de mourir, peur de perdre contrôle, peur de l'isolement, peur que les soins infirmiers soient inadéquats et peur de l'avenir.

- Y a-t-il des chances que notre fils meure au cours de la thérapie?

- J'ai tellement peur (sanglots). En quel état sera mon cerveau après la radiothérapie? Est-ce que je pourrai encore penser?

- Ca fait tellement longtemps que je suis ici avec toutes les infirmières; maintenant j'ai peur de rentrer à la maison.

- Toutes les infirmières ne semblent pas avoir les mêmes capacités.

- Nous avons tous peur de ce qui arrivera après la greffe. Une fois qu'elle est terminée, on ne sait pas à quoi s'attendre ou ce que nous réserve l'avenir. 
Thème deux : Le besoin continu d'information/d'éducation

- J'aurais aimé savoir que ce serait comme ça. Personne ne m'a dit que je serais si malade et si faible.

- J'ai besoin de savoir quoi faire quand j'irai à la maison; nous avons un nouveau bébé, et ma femme doit s'en occuper.

Thème trois : Le besoin de se sentir bien pris en charge par les membres de la famille et par le personnel infirmier

- Je suis si chanceuse d'avoir un bon mari. Il est si occupé à travailler et à se charger des tâches ménagères.

- Elle (la coordonnatrice) est si aimable. Elle prend toujours le temps de m'écouter et de répondre à mes questions.

- Certaines infirmières font leur travail, sans plus. Je ne crois pas qu'elles comprennent ce que nous vivons.

Thème quatre : Le besoin de continuité au niveau des soins entre l'établissement d'orientation, le centre de greffe et la collectivité

- Je ne crois pas que les médecins et les infirmières des différents hôpitaux communiquent entre eux. Personne ne semble savoir ce que fait l'autre groupe.

- Chaque jour, une nouvelle infirmière communautaire se présente et ne semble pas savoir ce dont j'ai besoin.

Thème cinq : Le désir de connaître les effets secondaires du traitement et la façon de gérer les situations de toxicité

- Les patients doivent savoir à quoi s'attendre afin de ne pas paniquer.

- Personne ne m'a dit que le Maxeran me rendrait nerveux.

- Je m'en vais à la maison alors que je prends du Neupogen; j'ai peur.

Thème six : Les connaissances des infirmières inspiraient confiance dans les soins

- Lors de la collecte des cellules souches, une infirmière est toujours à vos côtés. Vous n'êtes pas seul, et l'infirmière vous explique tout ce qui se passe. Vous ne vous sentez même pas malade. Je me sentais parfaitement bien à chaque prélèvement de cellules souches. J'avais le sentiment d'exercer un contrôle sur ce qui m'arrivait.

- Elle (la coordonnatrice) m'a fait sentir que tout irait bien. J'avais entièrement confiance en elle.

Thème sept : Les infirmières de l'établissement d'orientation et les infirmières en santé communautaire semblaient posséder moins de connaissances relatives aux greffes

- Les infirmières en santé communautaire et les infirmières de l'établissement d'orientation semblent en savoir moins au sujet des greffes. Ainsi, l'infirmière communautaire a téléphoné de chez moi à la pharmacie pour demander ce qu'elle devait utiliser pour irriguer la ligne intraveineuse. J'ai la sensation qu'elles ne savent pas comment s'occuper de mon tube. Leurs soins ne m'inspirent pas autant confiance.

- Les infirmières à mon hôpital admettent qu'elles ne peuvent pas répondre à certaines de mes questions concernant ce qui peut se passer durant la greffe.

L'analyse de la catégorie des infirmières a permis de cerner six thèmes.

Thème un : Les infirmières coordonnatrices sont conscientes de l'impact psychosocial de la greffe sur les patients et leur famille.

- Le processus de greffe est très complexe. Les patients sont souvent très loin de leur famille. Nous savons qu'ils ont peur. Ils se demandent s'ils vont mourir, s'ils retourneront un jour au travail, s'ils deviendront un fardeau financier.

- Ils attendent souvent longtemps après l'évaluation de l'admissibilité avant de débuter le traitement. Les patients craignent que la maladie progresse et qu'ils mourront avant le début du traitement.
Thème deux : Les infirmières coordonnatrices sont frustrées par leur rôle exhaustif et polyvalent.

- Nous fixons les rendez-vous pour les tests de diagnostic, nous faisons les arrangements pour les admissions à l'hôpital et les traitements de chimiothérapie dans les centres d'aiguillage et nous nous assurons que le traitement préliminaire a été suffisant. Nous devons faire énormément de travail administratif.

Thème trois : Temps limité pour l'enseignement aux patients/familles et pour le soutien offert par les coordonnateurs des interventions de greffe.

- Les patients font des séjours plus courts à l'hôpital en raison des améliorations technologiques et du financement limité destiné aux interventions de greffe.

- Nous n'avons pas le temps de voir les patients avant qu'ils rentrent chez eux ou même une fois qu'ils sont admis pour une greffe. Cela se doit en partie aux taux accrus d'orientation. Nous passons maintenant une plus grande proportion de notre temps à remplir nos fonctions d'infirmières de clinique externe, c'est-à-dire à faire des anamnèses et des évaluations.

- Notre trousse d'enseignement est désuète et elle contient trop de matériel. Mais nous n'avons pas le temps de la mettre à jour.

Thème quatre : Les coordonnateurs des interventions de greffe reconnaissent le besoin de défendre les intérêts des patients.

- Lors de l'évaluation, le spécialiste donne aux patients/familles une quantité considérable d'information. On peut voir qu'ils sont dépassés, et nous avons bien peu de temps par la suite pour répéter l'information ou pour répondre à leurs questions.

- Les patients sont envoyés chez eux trop tôt. Dans bien des cas, ils ne boivent pas assez et sont très faibles. Les patients doivent boire trois litres de liquide par jour et sont incapables de le faire.

Thème cinq : Les infirmières des centres de greffe reconnaissent les exigences en matière de connaissances et de compétences.

- Nous sommes occupées toute la journée à surveiller les températures élevées, à administrer des produits sanguins et des antibiotiques.

- Il est absolument nécessaire de surveiller les signes avant-coureurs de complications et d'agir immédiatement. L'état des patients peut très rapidement devenir septique.

- Cependant, nous avons presque suffisamment de temps pour faire notre travail.

Thème six : Les coordonnatrices des centres de greffe sont reconnues comme des expertes.

- Nous (infirmières coordonnatrices) recevons jusqu'à 70 appels par jour de la part de patients/familles qui ont besoin de soutien et d'être rassurés. Les patients ont confiance en ce que nous leur disons mais les infirmières communautaires pourraient fournir la même information.

- Nous (les coordonnatrices de centre d'accès aux soins communautaires) pouvons téléphoner au centre de greffe lorsque nous avons des questions.

\section{Résultats}

À partir de ces thèmes, les étudiantes-chercheuses ont cerné les besoins suivants des patients/familles qui subissent une GACSSP : soutien affectif/spirituel, éducation/information, continuité des soins, communication entre les fournisseurs de soins et enfin, infirmières bien informées et compétentes. De ces besoins, les étudianteschercheuses ont postulé que les connaissances, la communication, le soutien et les compétences techniques sont des éléments essentiels pour répondre aux besoins de cette population et pour améliorer la qualité des soins. 


\section{Connaissances}

Le nombre croissant d'orientations pour GACSSP, d'une part, et la tendance à accorder des congés précoces, d'autre part, mettent en évidence la nécessité pour les infirmières du centre d'aiguillage et les infirmières communautaires d'acquérir une solide compréhension du processus de greffe et de la gestion des soins complexes qui accompagnent de telles interventions. Il y a également un besoin de préparation et d'enseignement accrus pour les patients/familles afin de faciliter la prise de décisions, l'adaptation et la gestion des soins auto-administrés lors de l'aiguillage, après la greffe et après le congé.

\section{Communication}

Les patients, les membres de la famille et les infirmières des centres de greffe ont cerné le besoin d'une meilleure communication et d'une meilleure coordination des soins entre le centre de greffe, le centre d'orientation et la collectivité.

\section{Soutien}

L'impact psychologique considérable d'une GACSSP sur les patients/familles se poursuit tout au long du processus de greffe et s'intensifie au moment de l'aiguillage, après la greffe et au moment du congé. L'offre de renseignements exacts et cohérents, conjointement à un soutien psychologique et spirituel, devrait aider les patients/familles à mieux gérer la situation, ce qui contribuera à leur bien-être.

\section{Compétences}

L'évolution continuelle des tendances au niveau du traitement de GACSSP et de la gestion des soins complexes est telle que les infirmières ont constamment besoin de formation afin de tenir leurs compétences à jour et de se conformer aux normes de la pratique des soins infirmiers en oncologie. Vu que la GACSSP est une sousspécialité de l'oncologie, qui est elle-même une spécialité, de solides connaissances du processus et de la gestion des soins encouragera l'excellence dans les soins infirmiers en oncologie. En outre, les congés rapides exigent des patients/familles qu'ils acquièrent des compétences qui facilitent les soins auto-administrés, la gestion des symptômes et la prévention ou la détection précoce des complications afin de limiter les réadmissions à l'hôpital.

Un examen de la documentation actuelle continue de révéler que l'enseignement aux patients/familles, le soutien et la coordination des soins par des infirmières qui connaissent bien le processus de greffe constituent des éléments cruciaux de la gestion des soins pour cette population (Gillis et Donovan, 2001; Grant, Cooke, Bhatia et Forman, 2005; Kemp et Dickerson, 2002; Kohda et al., 2001).

\section{Conclusions}

Les résultats obtenus dans le cadre de cette recherche ont incité les chercheuses à mettre au point un livret d'enseignement pour les patients/familles afin de les aider à développer leurs capacités d'auto-soins une fois qu'ils ont obtenu leur congé du centre de soins tertiaires. Le livret contenait des renseignements sur les complications à court terme telles que les signes et symptômes découlant d'une chimiothérapie à forte dose et les actions appropriées qui s'imposent. Ces dernières portaient sur le moment judicieux d'entrer en contact avec l'oncologue local ou de se rendre à l'urgence la plus proche. Le livret contenait également une liste des intervenants de soins de santé et leur numéro de téléphone, une fiche récapitulative de données de laboratoire et de renseignements concernant les médicaments, et de l'information sur l'entretien d'un cathéter veineux central. Le livret abordait également des complications à long terme telles que la fatigue, les problèmes liés à la sexualité et les difficultés d'ordre social, et il offrait une liste de ressources communautaires utiles. Le livret était fait pour être distribué aux patients/familles au début du processus de greffe. Un second objectif de cet outil pédagogique était de fournir un moyen de communication permettant d'améliorer la coordination des soins entre les infirmières du centre de soins tertiaires, du centre d'aiguillage et des soins communautaires.

La mise au point d'un cadre de travail conceptuel qui propose une approche visant à améliorer la prestation des soins à cette population particulière était un autre résultat de la recherche. Le cadre de travail utilisait une approche systémique regroupant divers sous-systèmes ou parties interreliés et ouverts. Ensemble, ils déterminent la fonction totale du système. Au cœur du cadre de travail se trouve le soussystème patient/famille, soit le centre névralgique du système de prestation de soins. À l'intérieur du sous-système patient/famille se trouvent le sens subjectif du processus de greffe pour l'individu, les valeurs et les croyances, ainsi que le contexte physique, social, culturel, économique et politique de chaque être humain (modèle McMaster des soins infirmiers, 1995). Cette structure établit des liens entre trois sous-systèmes : le centre d'orientation, le centre de soins tertiaires et la collectivité. Ces sous-systèmes englobent le soussystème patient/famille et s'y superposent. En outre, ils présentent des variations internes selon la composition de l'équipe pluridisciplinaire, les services et les ressources. Ils s'inscrivent tous à l'intérieur d'une limite, soit le processus de la GACSSP. Le fonctionnement du système changera constamment selon le nombre de cas d'aiguillage, les progrès technologiques et la gestion des soins. Le maintien de l'équilibre à l'intérieur du système dépend du niveau de connaissances, de compétences, de soutien et de communication que possèdent ou dont font preuve les infirmières assignées aux soins des patients/familles qui subissent une GACSSP.

$\mathrm{Si}$ toutes les infirmières acquièrent les connaissances et compétences essentielles du processus de greffe et de la gestion des soins, elles : (a) comprennent les besoins de cette population et (b) dispensent de l'information et des soins biopsychosociaux et spirituels. Cela renforce les capacités des patients/familles d'auto-administrer leurs soins et allège ou minimise les complications tout au long du processus de greffe, ce qui a pour effet de promouvoir l'optimisation du bien-être. La communication coopérative entre tous les sous-systèmes mène à la continuité des soins et de l'information. Par conséquent, le rôle d'infirmière constitue la composante intégrative du système, la force régulatrice qui contribue à maintenir l'équilibre du système.

\section{Recommandations}

Les chercheuses ont proposé trois recommandations basées sur les résultats de recherche. La première était que les infirmières qui souhaitent pratiquer dans n'importe quelle phase de la GACSSP réussissent, à titre d'exigence minimale, un cours de certification sur les greffes de moelle épinière. Des programmes tels que celui de l'Université McMaster sur les soins en oncologie destinés aux adultes permettraient aux infirmières des centres de soins tertiaires et des centres d'aiguillage, de même qu'aux infirmières communautaires, d'acquérir une formation structurée optimale. Ce programme permettrait aux infirmières d'obtenir des postes en clinique dans des établissements spécialisés dans les interventions de moelle épinière et de GACSSP, et leur fournirait des expériences cliniques essentielles en oncologie. Les infirmières possédant une expérience et des connaissances du processus de greffe et de la gestion des soins pourraient offrir une formation continue et à jour à leurs pairs du centre d'aiguillage et des services communautaires. Cette stratégie exigerait une approche de gestion permettant le préceptorat. Deuxièmement, les chercheuses ont proposé que dans le centre de greffe, les rôles infirmiers soient améliorés en ce qui concerne la planification des congés. On a recommandé que les infirmières de la clinique externe d'oncologie offrent un enseignement à cet effet afin de faire en sorte que les patients/familles reçoivent une préparation et un enseignement à la fois adéquats et personnalisés avant leur congé. Les infirmières aborderaient l'information pertinente contenue dans le livret à la suite de chaque phase du processus de traitement. Cette discussion renforcerait l'enseignement aux patients/familles. 
Troisièmement, les chercheuses ont fait valoir que l'application du cadre de travail conceptuel et l'utilisation de la trousse d'enseignement au congé mèneraient à la prestation de soins de qualité axés sur le patient pour cette population particulière.

\section{Discussion}

L'approche phénoménologique s'est révélée être l'un des principaux points forts de ce projet de recherche. La phénoménologie examine le sens des expériences de vie et ne tente pas de faire de suppositions quant à la perception qu'ont les participants de leur situation. Cette approche met l'accent sur le caractère subjectif de l'expérience humaine et sur le contexte social dans lequel se produisent les événements (Polit et Hungler, 1997). Ainsi, la collecte de données auprès des patients, des membres de la famille et des infirmières en milieu naturaliste, $p$. ex. le centre de greffe, le domicile ou le bureau, mène à une compréhension des événements du point de vue du participant. En outre, les sources de données et la méthodologie de même que les « relèvements croisés » des chercheuses ont également contribué à la force de la recherche. On a interviewé des participants clés appartenant à trois catégories. Toutes les données ont été recueillies par deux chercheuses par le biais de l'observation, d'entretiens non structurés et de notes prises sur le terrain. La crédibilité des données s'est vue améliorée par la comparaison des observations du comportement dans une situation donnée avec la description narrative offerte par le participant, de même que par le recours à plus d'une personne pour collecter, analyser et interpréter les données. Le caractère confirmable des résultats s'est vu rehaussé par l'analyse thématique indépendante individuelle (Polit et Hungler, 1997).

L'étude avait cependant des limites. On a utilisé un échantillon accidentel, et deux patients avaient déjà subi une greffe autologue de la moelle épinière. D'autres facteurs qui ont limité les résultats de l'étude avaient à voir avec le processus d'entrevue : les entrevues n'étaient pas enregistrées,et des entrevues de validation n'ont pas été réaliées. L'enregistrement sonore des entrevues ou l'enregistrement complet de l'information des entrevues auraient rehaussé l'exactitude, la crédibilité et le caractère exhaustif des résultats. Les entrevues de suivi permettent aux chercheuses de s'assurer que l'interprétation est correcte et d'apporter des clarifications, au besoin (Berg, 1998). En outre, on peut alors obtenir plus de détails. Il est cependant difficile de déterminer la méthode la plus efficace de collecte de données durant les entrevues tout en maintenant une attitude humaine et empathique envers le participant. D'autres limites d'ordre méthodologique découlaient de l'absence d'un système de codage conçu au préalable pour l'analyse des données. Les deux chercheuses ont analysé le contenu en codant les concepts et les thèmes se dégageant des données recueillies dans chaque catégorie de patients. Bien que le système de codage n'était pas aussi détaillé et systématique que celui de Burnard (1991), il suivait plusieurs des mêmes stades d'analyse. Les chercheuses ont analysé les données indépendamment et ont comparé les résultats. Elles ont tenté de représenter les pensées et sentiments des participants d'une façon systématique et fidèle. Afin d'assurer le caractère généralisable des résultats, on devrait mener d'autres études dans lesquelles la sélection des participants se ferait par échantillonnage contrôlé dans un groupe de centres de soins tertiaires, de centres d'aiguillage et de cliniques communautaires choisis au hasard, et on devrait choisir des participants ne subissant aucune intervention autre qu'une GACSSP.

\section{Résumé}

Les résultats de cette recherche ont été diffusés dans le cadre de la conférence annuelle de l'ACIO à Regina en 1998, sous la forme d'une présentation d'affiches dirigée. On a expliqué au moyen de graphiques le modèle de soins proposé pour cette population, et les participants ont pu examiner le livret éducatif. Après la conférence, la coordonnatrice d'un centre de soins tertiaires du Sud de l'Ontario s'est servie du livret en tant qu'outil pédagogique auprès de la population de patients subissant une GACSSP. Étant donné que la documentation actuelle continue de souligner la hausse dans cette population, des besoins de gestion des soins aux patients traités en clinique externe, les recommandations des étudiantes-chercheuses en matière de formation infirmière, d'utilisation du cadre de travail conceptuel pour la prestation des soins et d'utilisation de matériel éducatif actualisé pour les patients sont possiblement encore pertinentes aujourd'hui. Les étudiantes ont reçu le premier Prix national annuel AMGEN/ACIO pour l'innovation dans l'enseignement aux patients/familles. On mène actuellement des recherches sur les caractéristiques des soins infirmiers et leur incidence sur la capacité des patients/familles subissant une GACSSP à auto-administrer leurs soins .

\section{Références}

Association canadienne des infirmières en oncologie (2001). Normes de soins, rôles infirmiers en oncologie et compétences relatives aux rôles infirmiers. CANO Nursing Education Project.

Berg, B. (1998). Qualitative research methods for the social sciences (3rd ed.). Boston: Allyn and Bacon.

Burnard, P. (1991). A method of analyzing interview transcripts in qualitative research. Nurse Education Today, 11, 461-466.

Coleman, E., Coon, S., Mattox, S., \& O’Sullivan, P. (2002, December). Symptom management and successful outpatient transplantation for patients with multiple myeloma. Cancer Nursing, 26(6), 452-460.

Grant, M., Cooke, L., Bhatia, S., \& Forman, S. (2005, January). Discharge and unscheduled re-admissions of adult patients undergoing hematopoietic stem cell transplantation: Implications for developing nursing interventions [Electronic version]. Oncology Nursing Forum, 32(1), 1-12. Retrieved February 20, 2005, from http://www.ons.org/publications/journals/ONF/ Volume32/Issue1/320130.asp

Gillis, T., \& Donovan, E. (2001). Rehabilitation following bone marrow transplantation. Cancer, 92(Suppl. 4), 998-1007.

Hurley, C. (1997). Ambulatory care after bone marrow or peripheral blood stem cell transplantation. Clinical Journal of Oncology Nursing, 1(1), 19-21.
Johns, A. (1998). Overview of bone marrow and stem cell transplantation. Journal of Intravenous Nursing, 21(6), 356360.

Kemp, J., \& Dickerson, J. (2002). Interdisciplinary modular teaching for patients undergoing progenitor cell transplantation. Clinical Journal of Oncology Nursing, 6(3), $1-4$.

Kohda, K., Sakamaki, S., Matsunaga, T., Juga, T., Fujimi, A., Konuma, Y., et al. (2001). Long-term survival and late onset complications of cancer patients treated with high-dose chemotherapy followed by autologous peripheral blood stem cell transplantation. International Journal of Hematology, 73, 251257.

Polit, D., \& Hungler, B. (1997). Essentials of nursing research: Methods, appraisal, and utilization (4th ed.). New York: Lippincott.

Poloquin, C. (1997). Overview of bone marrow and peripheral blood stem cell transplantation. Clinical Journal of Oncology Nursing, 1(1), 11-17.

Schulmeister, L., Quiett, K., \& Mayer, K. (2005). Quality of life, quality of care, and patient satisfaction: Perceptions of patients undergoing outpatient autologous stem cell transplantation. Oncology Nursing Forum, 32(1), 57-67. 